\title{
Metastatic Adenocarcinoma of right supraclavicular lymph nodes from primary ovarian cancer: a rare presentation
}

\begin{abstract}
Background: Lymph-node metastases to the right supraclavicular lymph nodes (RSCLNs) in ovarian malignancies are very rare occurrences. The majority of RSCLN metastases normally originate from primary neck, lung, and esophagus malignancies. A literature review revealed that only 2 cases of ovarian metastases to the RSCLNs have been reported.

Case: A 28-year-old, single nulliparous woman presented to a local tertiary hospital with a 2month history of progressive abdominal distension. This was associated with a loss of appetite and a marked weight loss. She had no significant medical illnesses or family history of malignancies. Examination revealed a nontender palpable lymph node in the right supraclavicular region, measuring $4 \times 3 \mathrm{~cm}$. No other lymph nodes were palpable at the left supraclavicular or cervical areas. There was also gross ascites. Other examination results were unremarkable. A computed tomography (CT) scan showed an RSCLN mass and complex solidcystic masses arising from the pelvis. Fine-needle aspiration (FNA) biopsy of the RSCLN was performed, revealing a metastatic adenocarcinoma with features compatible with serous adenocarcinoma of the ovary.

Results: This patient was treated successfully with neoadjuvant chemotherapy that was followed by a total abdominal hysterectomy, and bilateral salpingo-oophorectomy, omentectomy, and appendectomy. She was alive with no recurrences at a 1-year regular followup.

Conclusions: The rarity of RSCLN metastases in ovarian cancer might incline clinicians to neglect the presence of RSCLN enlargement during examinations. Thus, clinicians should examine both left and right SCLNs thoroughly in women suspected of having gynecologic malignancies.
\end{abstract}

Keyword: Case study; Fine-needle aspiration biopsy; Metastasis; Ovarian cancer; Right supraclavicular lymph node 